\title{
Do neurosurgeons subscribe to the guideline lumbosacral radicular syndrome?
}

\author{
P.A.J. Luijsterburg ${ }^{\text {a,* }}$, A.P. Verhagen ${ }^{\text {a }}$, S. Braak ${ }^{\text {a }}$, C.J.J. Avezaat ${ }^{b}$, B.W. Koes ${ }^{\text {a }}$ \\ a Department of General Practice, Erasmus MC, University Medical Center Rotterdam, 3000 DR Rotterdam, The Netherlands \\ ${ }^{\mathrm{b}}$ Department of Neurosurgery, Erasmus MC, University Medical Center Rotterdam, 3000 DR Rotterdam, The Netherlands
}

Received 9 October 2003; received in revised form 2 February 2004; accepted 6 February 2004

\begin{abstract}
Background: This study presents a survey of the opinion of neurosurgeons on the multidisciplinary clinical guideline 'lumbosacral radicular syndrome'. The aim was to describe to what extent neurosurgeons in the Netherlands endorse the content of this guideline. The guideline was issued in 1996 by the Netherlands Institute of Quality Health Care and this is the first attempt to evaluate the implementation of this guideline. Methods: All active neurosurgeons $(n=92)$ in the Netherlands were invited to complete a questionnaire investigating to what extent they agree with the 26 recommendations in the guideline 'lumbosacral radicular syndrome'. The results are represented in frequencies (\%) in order to express the magnitude of their consent or dissent with the recommendations. Results: Overall, $75 \%$ of the neurosurgeons responded and, of these, $94 \%$ agreed (at least partially) with the content of the guideline. Of the 26 recommendations in the guideline, seven were not fully endorsed by the neurosurgeons. Three of these seven recommendations may need revision based on newly published data. Conclusion: This survey shows that almost all neurosurgeons subscribed (at least partially) to the multidisciplinary LRS guideline. Therefore, one important aspect of the implementation process has been fulfilled, i.e. acceptance of the content of the guideline.
\end{abstract}

(C) 2004 Published by Elsevier B.V.

Keywords: Lumbosacral radicular syndrome; Neurosurgeon; Guideline; Sciatica

\section{Introduction}

The lumbosacral radicular syndrome (LRS), also known as 'sciatica', is a disorder with radiating pain in one or more lumbar or sacral dermatomes, and can be accompanied with phenomena associated with nerve root tension or neurological deficits [1-4]. LRS is mostly caused by a prolapsed disc, but other causes can include spinal or lateral recess stenosis and tumours or radiculitis $[4,5]$. The incidence of LRS in the Netherlands is estimated between 60,000 and 75,000 [5]. In the Netherlands the neurosurgeons generally perform surgery in patients with LRS caused by a prolapsed disc.

A multidisciplinary clinical practice guideline for LRS was issued in 1996 by the Netherlands Institute of Quality Health Care [3]. This guideline (as far as possible evidence-based) is a consensus between 12 (para)medical professions involved in the management of patients with

\footnotetext{
* Corresponding author. Tel.: +31-10-463-2121; fax: +31-10-463-2127.

E-mail address: p.luijsterburg@erasmusmc.nl (P.A.J. Luijsterburg).
}

LRS. The guideline presents 26 recommendations (see Appendix A) which serve to guide physicians in their management of patients with LRS.

Clinical guidelines are developed to improve quality of health care and to foster evidence-based decision making [6-9], but their recommendations must be implemented to achieve the desired outcomes. In order to evaluate the implementation of a particular guideline, it is important to know whether the profession concerned accepts the content of the guideline [7]. Therefore, this study investigated to what extent neurosurgeons in the Netherlands endorse the content of the LRS guideline.

\section{Materials and methods}

In June 2001, a questionnaire about the LRS guideline was mailed to all 92 active neurosurgeons associated with the Netherlands Society of Neurosurgeons, together with a letter of recommendation from the chairman of the Society. Reminders were sent after 1 and 2 months. 
The questionnaire addressed the following: (1) neurosurgeons' characteristics including age, gender, years of working experience and their type of neurosurgery centre (i.e. university/non-university); (2) to what extent they are acquainted with the content of the multidisciplinary guideline, with answer options: (almost) entirely, partially, and (almost) not; (3) to what extent they subscribe to the content of the guideline, with answer options: (almost) entirely, partially, and (almost) not; and (4) to what extent they agree with each of the 26 recommendations in the guideline, each recommendation had the answer option: (almost) entirely, partially, and (almost) not. All answers could be explained with an explanation if required. In addition, because two of the 26 recommendations concern general practitioners (GPs) and because GPs have their own LRS guideline [2], neurosurgeons were also asked about their acquaintance with the GPs' guideline for LRS.

Descriptive statistics were used to present the frequencies of the neurosurgeons' agreement/disagreement with the multidisciplinary guideline; all frequencies are based on the total number of neurosurgeons. All variables used to describe the neurosurgeons' characteristics are presented as the mean and standard deviation (S.D.) if they are normally distributed, and by the median and interquartile range (IQR) if they are not normally distributed. We decided that any of the 26 recommendations in the questionnaire was debatable when $60 \%$ or less of the neurosurgeons did not agree (almost) entirely with a particular recommendation. All data were coded and analyzed using the statistical package SPSS for Windows (version 10.0.7).

\section{Results}

\subsection{Neurosurgeons' characteristics}

Of the 92 invited neurosurgeons, $69(75 \%)$ returned the questionnaire. Because three of the responding neurosurgeons stated that they did not treat LRS patients, data from 66 respondents were included in the analysis. Reasons for non-response are unknown because the questionnaires were returned anonymously. The median age of the neurosurgeons was 45 (IQR 40-55) years, 9\% were female, and the median work experience was 12 (IQR 5-22) years. The neurosurgeons estimated to manage a median of 60 LRS patients (IQR 25-92) during a 3-month period. The median number of patients with LRS who underwent surgery was estimated to be 30 (IQR 15-60) patients in a 3-month period.

\subsection{Subscribing to the LRS guideline}

The neurosurgeons were asked if they were acquainted with the content of the multidisciplinary guideline and to what extent they subscribe to the guideline. Table 1 shows that $91 \%$ are acquainted (at least partially) with the content of the guideline, and that $94 \%$ subscribe (at least partially)
Table 1

Neurosurgeons' responses to questions about the multidisciplinary LRS guideline and the LRS guideline issued by the Dutch College of General Practitioners (GPs' guideline)

\begin{tabular}{lc}
\hline & Neurosurgeons $n(\%)$ \\
\hline Acquainted with the content of the guideline \\
(Almost) entirely & $42(63.5)$ \\
Partially & $18(27.5)$ \\
(Almost) not at all & $6(9.0)$ \\
Missing answers & $0(0.0)$ \\
Subscribe to the guideline & \\
(Almost) entirely & $24(36.5)$ \\
Partially & $38(57.5)$ \\
(Almost) not at all & $0(0.0)$ \\
Missing answers & $4(6.0)$ \\
Acquainted with the content of the GPs' guideline \\
(Almost) entirely & $15(22.5)$ \\
Partially & $18(27.5)$ \\
(Almost) not at all & $33(50.0)$ \\
Missing answers & $0(0.0)$ \\
\hline
\end{tabular}

$n=$ number of neurosurgeons.

to the guideline. In contrast, only $50 \%$ were acquainted with the LRS guideline issued by the Dutch College of General Practitioners.

\subsection{Recommendations}

The Appendix A lists the 26 recommendations in the multidisciplinary LRS guideline. Table 2 shows to what extent the neurosurgeons agree/disagree with these recommendations.

Over $60 \%$ of the neurosurgeons completely agreed with 19 of the 26 recommendations. The remaining seven recommendations, which were endorsed by less than $60 \%$ of the neurosurgeons, are discussed here.

1. Arguments against recommendation 12 (The GP can perform clinical diagnostics and treatment in most LRS-patients) were: 'depends on the seriousness of the complaints $(n=2)$ ', 'expert report by the GP is questionable, knowledge of LRS is often insufficient $(n=6)^{\prime}$, and 'GPs' referral to a specialist is usually late $(n=2)$ '.

2. Arguments against recommendation 17 (The most important indication for surgery in prolapsed disc is severe radicular pain and not the sensory deficits, except for the cauda equina syndrome) were: 'the presence of neurological deficits can be an additional reason to perform surgery $(n=2)$ ', and 'as well as severe suffering an important indication for surgery $(n=17)$ '.

3. Arguments against recommendation 19 (After 6 weeks the GP should discuss the option of surgery with the patient with LRS when there is no clear improvement of the complaints) were: ' 6 weeks is too long $(n=8)$ ', and 'depending on the seriousness of the complaints and level of neurological deficits surgery must be considered sooner $(n=5)$ '. 


\section{Table 2}

Neurosurgeons' ( $n=66$ ) level of agreement/disagreement (frequencies) with the 26 recommendations in the multidisciplinary LRS guideline

\begin{tabular}{|c|c|c|c|c|}
\hline Recommendation & $\begin{array}{l}\text { (Almost) } \\
\text { entirely }(\%)\end{array}$ & $\begin{array}{l}\text { Partially } \\
(\%)\end{array}$ & $\begin{array}{l}\text { (Almost) } \\
\text { not }(\%)\end{array}$ & $\begin{array}{l}\text { Missing } \\
(\%)\end{array}$ \\
\hline 1 & 96 & 1 & 1 & 2 \\
\hline 2 & 82 & 17 & 0 & 1 \\
\hline 3 & 73 & 21 & 5 & 1 \\
\hline 4 & 89 & 9 & 0 & 2 \\
\hline 5 & 73 & 21 & 5 & 1 \\
\hline 6 & 68 & 17 & 11 & 4 \\
\hline 7 & 70 & 20 & 6 & 4 \\
\hline 8 & 64 & 20 & 9 & 7 \\
\hline 9 & 64 & 23 & 9 & 3 \\
\hline 10 & 35 & 41 & 18 & 6 \\
\hline 11 & 71 & 21 & 5 & 3 \\
\hline 12 & 55 & 33 & 12 & 0 \\
\hline 13 & 53 & 32 & 14 & 1 \\
\hline 14 & 82 & 9 & 6 & 2 \\
\hline 15 & 74 & 21 & 2 & 3 \\
\hline 16 & 83 & 14 & 3 & 0 \\
\hline 17 & 41 & 39 & 15 & 5 \\
\hline 18 & 6 & 27 & 59 & 7 \\
\hline 19 & 58 & 32 & 6 & 4 \\
\hline 20 & 55 & 26 & 15 & 4 \\
\hline 21 & 91 & 3 & 2 & 4 \\
\hline 22 & 76 & 14 & 5 & 5 \\
\hline 23 & 79 & 18 & 2 & 1 \\
\hline 24 & 65 & 29 & 6 & 0 \\
\hline 25 & 86 & 14 & 0 & 0 \\
\hline 26 & 65 & 30 & 0 & 5 \\
\hline
\end{tabular}

4. Arguments against recommendation 20 (There is no evidence that the prognosis of paresis improves by surgical intervention) were: 'experience has shown that muscle strength in the leg does improve after surgery $(n=7)$ ', and 'has not yet been evaluated, so it can be an indication $(n=5)^{\prime}$.

Finally, the neurosurgeons reported that new evidence was available that no longer supported three of the recommendations:

- Recommendation 10 (neurophysiological examination can provide additional information about the location and severity of the nerve root damage, when radiological findings are not in accordance with clinical findings) because: 'the additional information from a neurophysiological examination is poor $(n=9)$ '.

- Recommendation 13 (conservative treatments are not sufficiently investigated to draw conclusions regarding their effectiveness) because: 'bed rest, traction and psychotherapy have been examined and are demonstrated not to be useful $(n=16)^{\prime}$.

- Recommendation 18 (chemonucleolysis is proven effective for LRS caused by prolapsed disc; the results after 1 year correspond with the results of surgery) because: 'indication is limited, chemonucleolysis is not effective $(n=8)$ ', 'percentage of patients with complaints after chemonucleolysis is higher than after surgery $(n=6)$,
Table 3

Neurosurgeons' $(n=66)$ recommended timing of surgery for patients with LRS caused by a prolapsed disc

\begin{tabular}{lr}
\hline Surgery after & $\%^{\mathrm{a}}$ \\
\hline 2-6 weeks & 17 \\
6 weeks & 18 \\
6-8 weeks & 5 \\
8 weeks & 14 \\
8-12 weeks & 9 \\
12 weeks & 15 \\
12-16 weeks & 6 \\
\hline
\end{tabular}

a Missing values $16 \%$.

and 'chemonucleolysis is effective but less effective than surgery $(n=3)$ '.

\subsection{Indication for and timing of surgery}

The questionnaire asked the neurosurgeons: 'What, in your opinion, is the indication for surgery in patients with LRS caused by a prolapsed disc?' The neurosurgeons reported eight indications: the cauda equina syndrome (68\%), long-term and disabling pain (67\%), progressive paresis (33\%), radiological findings in accordance with clinical signs $(15 \%)$, patients' wishes (15\%), recurrences $(11 \%)$, acute paresis (9\%), and persisting complaints (6\%).

They were also asked: 'At what point of time after the onset of an LRS episode do you recommend surgery for a patient with LRS caused by a prolapsed disc with pain and no neurological deficits?' Table 3 gives the neurosurgeons' answers (range 2-16 weeks) to this question.

About half of the neurosurgeons (54\%) preferred to wait for $2-8$ weeks and the remainder preferred to wait for $8-16$ weeks before performing surgery.

\section{Discussion}

An important condition for the implementation of any guideline is the extent to which a profession agrees with the content of the guideline $[7,10]$. This survey shows that most (94\%) of the participating neurosurgeons agrees (at least partially) with the content of the multidisciplinary guideline for the management of LRS. The neurosurgeons endorsed with 19 of the 26 recommendations in the guideline; the remaining seven recommendations were accepted by less than $60 \%$ of the neurosurgeons. Recommendations 17, 19, and 20 are still being debated because there are (as yet) no convincing data available. Due to a difference in viewpoint between neurosurgeons and GPs, less than $60 \%$ of the neurosurgeons agreed with recommendation 12 (The GP can perform clinical diagnostics and treatment in most LRS patients). This difference may be due to the fact that $50 \%$ of the neurosurgeons were not aware of the content of the LRS guideline issued by the Dutch College of General Practitioners. For recommendations 10,13 , and 18 the neuro- 
surgeons reported that new evidence invalidated these three recommendations.

In spite of the high response $(75 \%)$ to the questionnaire some selection bias may have occurred. For example, because the questionnaire was returned anonymously, we have no way of determining the reasons for non-response. Although it is possible that our results under- or over-estimate acceptance of the guideline by neurosurgeons, we cannot confirm this.

Because the questionnaire was specifically developed for this study based on the content of the LRS guideline, no data on the reliability and validity of this questionnaire are available. Also we arbitrarily selected a cut-off point of $60 \%$ or less as a measure of whether or not a recommendation was endorsed.

In the Netherlands, patients with the lumbosacral radicular syndrome are not only operated by neurosurgeons. Also orthopedic surgeons perform this operation, but only in a minority of patients $(17 \%)$. The results of this study cannot be generalized towards the orthopedic surgeons in the Netherlands.

The timing of sugery for a patient with LRS caused by a prolapsed disc ranged from 2 to 16 weeks (Table 3 ). Half of the neurosurgeons preferred to wait less than 8 weeks, and the others preferred to wait more than 8 weeks. Recommendation 16 of the guideline indicates a waiting period of 4-8 weeks.

The LRS guideline of the Dutch College of General Practitioners recommends referral to a specialist when there is no improvement after $6-8$ weeks. The literature provides no evidence for the most optimal timing of surgery.

The adherence towards the guideline LRS in neurosurgeons' daily practice was not evaluated in this study. In the Netherlands, we do not know to what extent the neurosurgeons actually follow the recommendations of the guideline LRS. Here we only evaluated their altitude towards the guideline because without a positive attitude, implementation becomes difficult.

\section{Conclusion}

This study shows that neurosurgeons largely subscribe to the guideline for the management of LRS. The next important step is to investigate the neurosurgeons' actual management of LRS patients compared with the guideline recommendations. Nevertheless, guidelines need to be regulary updated to remain useful to clinicians. The multidisciplinary clinical guideline for the management of LRS was issued in 1996. The present study, 6 years later, shows that neurosurgeons consider that three recommendations need to be updated based on new evidence. Shekelle et al. estimated that guidelines should be reassessed for validity every 3 years [11]. Therefore, more studies are needed on the management of LRS in order to update the guidelines for this syndrome.

\section{Appendix A. Recommendations in the multidisciplinary clinical guideline for the management of LRS}

1. LRS is characterized by radiating pain in one or more lumbo or sacral dermatomes, with or without other radicular symptoms.

2. LRS is often caused by a prolapsed disc, but is also caused by a spinal or resessus stenosis, or a combination of these.

3. LRS cannot be explained by mechanical compression of a nerve root only.

4. Many radiological abnormalities in the lumbar spine are not associated with nerve root compression or pain.

5. The test of Lasègue is the most valid and reliable test in acute LRS due to prolapsed disc. The test of Lasègue is often negative in neurological claudicatio.

6. There is no indication for routine X-rays of the lumbar spine in acute LRS.

7. MRI or CT-scan is only needed when surgery is considered, or if the results could have other therapeutical consequences.

8. MRI and CT-scan both have a high sensitivity for detecting a prolapsed disc and a low specificity: it is often impossible to distinguish between a prolapsed disc that causes the LRS and an accidental finding on the basis of imaging alone.

9. In radiological imaging of LRS, MRI is preferred; CT-scan is a good alternative. If uncertainty of nerve root compression persists caudography is indicated.

10. Neurophysiological examination can provide additional information about the location and severity of the nerve root damage, when radiological findings are not in accordance with clinical findings.

11. LRS is often self-limiting. The results of conservative treatments and surgery are similar in the long term (4-10 years), i.e. for those patients who do not undergo surgery because of progressive paresis or cauda equina syndrome.

12. The GP can perform clinical diagnostics and treatment in most LRS patients. Referral to a specialist is only useful when the GP is not sure about the diagnosis or considers surgical intervention.

13. Conservative treatments (e.g. bed rest, traction, physiotherapy, and manipulation) are not sufficiently investigated to draw conclusions regarding their effectiveness.

14. The statement that strict bed rest (toilet visits and showering not permitted) is more effective in LRS due to prolapsed disc than liberal bed rest (toilet visits and showering permitted) is not based on prospective RCTs.

15. Effectiveness of 'back-schools' for LRS has not yet been investigated, neither for treatment, nor for prevention. 
16. A severe LRS, persisting for 4-8 weeks with no improvement, is an indication for radiological examination possibly followed by surgical intervention. If there is improvement, a longer 'wait and see' policy is possible.

17. The most important indication for surgery in prolapsed disc is severe radicular pain and not the sensory deficits, except for the cauda equina syndrome.

18. Chemonucleolysis is proven effective for LRS caused by a prolapsed disc; the results after one year correspond with the results of surgery.

19. After 6 weeks the GP should discuss the option of surgery with the LRS patient when there is no clear improvement of the complaints.

20. There is no evidence that the prognosis of paresis improves by surgical intervention. Therefore, a light or moderate paresis is not an absolute indication for surgery.

21. Cauda equina syndrome caused by lumbar disc prolapse is an absolute indication for rapid surgery.

22. Percutaneous nucleotomy and percutaneous laser therapy are not evidence-based treatments for LRS caused by disc prolapse.

23. There are no proven effective programs of treatment for primary or secondary prevention of LRS.

24. Advice not to work during and after the treatment of LRS, even in demanding jobs, should be given cautiously. This could delay rehabilitation.

25 . The treating surgeon is responsible for medical care before and after surgery.

26. There are strong indications that psychological, social, and financial factors play an important role in the development of persisting LRS (and the related disability).

\section{References}

[1] Ostelo RWJG, de Vet HCW, van Tulder MW, Kerckhoffs MR, Leffers P, Wadell G. Rehabilitation after lumbar disc surgery (protocol for a cochrane review), The Cochrane Library, 2002.

[2] Smeele IJM, Van den Hoogen JMM, Mens JMA, Chavannes AW, Faas A, Koes BW, et al. [NHG-guideline Lumbosacral Radicular Syndrome] NHG-Standaard Lumbosacraal Radiculair Syndroom. Huisarts en Wetenschap 1996;39:78-89.

[3] Stam J. [Consensus in diagnosing and treatment of the lumbosacral radicular syndrome] Consensus over diagnostiek en behandeling van het lumbosacrale radiculaire syndroom. Nederlands Tijdschrift Voor Geneeskunde 1996;140:2621-7.

[4] Weber H, Holme I, Amlie E. The natural course of acute sciatica with nerve root symptoms in a double-blind placebo-controlled trial evaluating the effect of piroxicam. Spine 1993;18:1433-8.

[5] Health Council of the Netherlands. Management of the lumbosacral radicular syndrome (sciatica). The Hague: publication no. 1999/1918, 1999.
[6] Grimshaw JM, Russell IT. Effect of clinical guidelines on medical practice: a systematic review of rigorous evaluations. The Lancet 1993;342:1317-22.

[7] Grol R, Wensing M. [Implementation. effective change in patient care.] Implementatie. effectieve verandering in de patiëntenzorg. second ed. Maarssen: Elsevier gezondheidszorg, 2001.

[8] Hayward RS, Wilson MC, Tunis SR, Bass EB, Guyatt G. Users' guides to the medical literature. VIII. How to use clinical practice guidelines. A. Are the recommendations valid? the evidence-based medicine working group. JAMA 1995;274:570-4.

[9] Miilunpalo S, Toropainen E, Moisio P. Implementation of guidelines in primary health care: a challenge for the municipal health centres in Finland. Scand J Prim Health Care 2001;19:227-31.

[10] Grol R. Personal paper: beliefs and evidence in changing clinical practice. BMJ 1997;315:418-21.

[11] Shekelle PG, Ortiz E, Rhodes S, Morton SC, Eccles MP, Grimshaw $\mathrm{JM}$, et al. Validity of the agency for healthcare research and quality clinical practice guidelines: how quickly do guidelines become outdated? JAMA 2001;286:1461-7. 